\title{
A STUDY OF DISCONTINUOUS GALERKIN METHODS FOR THIN BENDING PROBLEMS
}

\author{
N.T. Dung, G.N. Wells \\ Faculty of Civil Engineering and Geosciences, Delft University of Technology \\ P.O. Box 5048, 2600 GA Delft \\ e-mail: n.t.dung@citg.tudelft.nl, g.n.wells@ tudelft.nl
}

Keywords: Plates, thin bending, discontinuous Galerkin methods, stability condition.

\begin{abstract}
Various continuous/discontinuous Galerkin formulations are examined for the analysis of thin plates. These methods rely on weak imposition of continuity of the normal slope across element boundaries. We draw here upon developments in discontinuous Galerkin methods for second-order elliptic equations, for which several unconditionally stable methods are known, and present continuous/discontinuous Galerkin formulations for bending problems inspired by these methods. For each approach, benchmark simulations have been performed and compared. Also, conclusions are drawn on to the computational efficiency of the different methods.
\end{abstract}




\section{INTRODUCTION}

Classical thin bending theories have posed significant challenges for finite modeling element due to the fourth-order nature of the governing equations. The construction of conforming, or robust nonconforming, finite element formulations for two-dimensional problems is problematic. Given these difficulties, the majority of finite element models for bending problems in commercial codes are based on the Reissner-Mindlin theory. Formulations based on the Reissner-Mindlin theory circumvent the need for $C^{1}$ basis functions, at the expense of other difficulties, such as stability and locking.

Inspired by works on discontinuous Galerkin methods for elliptic problems, Engel et al. [1] developed a formulation for fourth-order elliptic problems which exploited standard $C^{0}$ finite element basis functions while maintaining consistency and stability (the latter being conditional upon the choice of a sufficiently large penalty term). The method introduced inter-element boundary integrals in order to enforce continuity of the normal derivative across element boundaries in a weak sense. The method has also been used for modelling strain-gradient dependent damage models [2, 3] and has been analysed by Brenner and Sung [4].

The method presented by Engel et al. [1] resembles the interior penalty method (see [5, 6]), and inherits its most significant drawback of conditional stability. It is not possible to quantify a priori how large the penalty term must be to ensure stability, an overly large penalty term has a negative impact on accuracy (see [4]) and may induce numerical difficulties. Here, we draw upon discontinuous Galerkin methods for second-order problems, for which methods are known in which required penalty parameter to ensure stability can be determined a priori, an overview of which can be found in [6]. We present here an extension of a particular method, due to Brezzi et al. [7], for thin bending problems and illustrate its performance.

To introduce the methods, the considered bending problem is formalised in Section 2. In Section 3, the interior-penalty based formulation for a plate is summarized, and the new method for plates is presented. The numerical implementation and efficiency issues are presented in Section 4, and the performance is examined by observing the convergence rates for a benchmark problem in Section 5. The results and some outstanding issues are summarised in Section 6.

\section{STRONG FORM OF THIN BENDING PROBLEMS}

Consider a flat polygonal plate that has a mid-surface denoted by $\Omega$ and thickness $t$. The boundary of $\Omega$ is denoted by $\Gamma=\partial \Omega$, and is partitioned such that $\overline{\Gamma^{w} \cup \Gamma^{Q}}=\overline{\Gamma^{\theta} \cup \Gamma^{M}}=\Gamma$ and $\Gamma^{w} \cap \Gamma^{Q}=\Gamma^{\theta} \cap \Gamma^{M}=\emptyset$. A Cartesian axis system $x, y, z$, in which the axis $z$ is normal to the mid-surface is adopted. Hereinafter, let $\alpha, \beta, \delta$ and $\gamma$ are indices denoting two directions $x$ and $y$, and $n_{\alpha}$ and $s_{\alpha}$ denote the unit outward normal vector and the unit tangent vector on boundaries, respectively.

The strong form can be declared as follows: given the distributed force $F$, the transverse displacement, rotation, moment, and transverse force boundary conditions, denoted by $g^{w}, g^{\theta}$, $M$, and $Q$, respectively, find the transverse displacement $w$ such that 


$$
\begin{aligned}
& m_{\alpha \beta, \alpha \beta}=F \quad \text { in } \Omega, \\
& w=g^{w} \quad \text { on } \Gamma^{w}, \\
& w_{, \alpha} n_{\alpha}=g^{\theta} \quad \text { on } \Gamma^{\theta}, \\
& m_{\alpha \beta} n_{\beta} n_{\alpha}=M \quad \text { on } \Gamma^{M}, \\
& -m_{\alpha \beta, \beta} n_{\alpha}-\left(m_{\alpha \beta} n_{\beta} s_{\alpha}\right)_{, s}=Q \quad \text { on } \Gamma^{Q} \text {, }
\end{aligned}
$$

where

$$
m_{\alpha \beta}=c_{\alpha \beta \gamma \delta} w_{, \gamma \delta},
$$

and $c_{\alpha \beta \gamma \delta}$ is a fourth order constitutive tensor. In the isotropic case, it is defined as:

$$
\begin{aligned}
c_{\alpha \beta \gamma \delta} & =\frac{t^{3}}{12}\left\{\mu\left(\delta_{\alpha \gamma} \delta_{\beta \delta}+\delta_{\alpha \delta} \delta_{\beta \gamma}\right)+\bar{\lambda} \delta_{\alpha \beta} \delta_{\gamma \delta}\right\}, \\
\bar{\lambda} & =\frac{E^{2}}{2(1+\nu)\left(1-\nu^{2}\right)}, \\
\mu & =\frac{E}{2(1+\nu)},
\end{aligned}
$$

where $E$ is Young's modulus, $\nu$ is Poisson's ratio, and $\delta_{\alpha \beta}$ is Kronecker's delta.

\section{DISCONTINUOUS GALERKIN FORMULATIONS FOR THIN BENDING PROB- LEMS}

Consider a partition $\mathcal{P}^{h}$ of the domain $\Omega$ into $n$ elements $E_{i}, i=1 \rightarrow n$, such that $\bigcup_{i=1}^{n} E_{i}=$ $\Omega$ ( $\Omega$ does not contain element edges). The union of all interior element edges is denoted by $\Gamma^{0}=\bigcup_{i=1}^{n} \partial E_{i} \backslash \partial \Omega$. The spaces of trial and weight functions are defined as follows:

$$
\begin{aligned}
& W^{h}=\left\{w^{h} \in H^{1}(\Omega):\left.w^{h}\right|_{E_{i}} \in P^{k}\left(E_{i}\right) \forall E_{i} \in \mathcal{P}^{h},\left.w^{h}\right|_{\Gamma^{w}}=g^{w}\right\}, \\
& \bar{W}^{h}=\left\{\bar{w}^{h} \in H^{1}(\Omega):\left.\bar{w}^{h}\right|_{E_{i}} \in P^{k}\left(E_{i}\right) \forall E_{i} \in \mathcal{P}^{h},\left.\bar{w}^{h}\right|_{\Gamma^{w}}=0\right\},
\end{aligned}
$$

where $P^{k}\left(E_{i}\right)$ are the standard finite element shape functions of degree $k$.

For two elements $E_{1}, E_{2} \in \mathcal{P}^{h}$, sharing an interior edge $e$ (see Figure 1), $n_{\alpha}$ denotes the normal vector on $e$ that points from $E_{1}$ to $E_{2}$. Let $a_{i}$ denote an arbitrary function with $a_{1}$ the value on element one and $a_{2}$ the value on element two. The jump $\llbracket a \rrbracket$ and the average $\langle a\rangle$ of $a_{i}$ on $e$ are defined as follows:

$$
\begin{aligned}
& \llbracket a \rrbracket=a_{1}-a_{2}, \\
& \langle a\rangle=\frac{1}{2}\left(a_{1}+a_{2}\right) .
\end{aligned}
$$

On an exterior edge, $n_{\alpha}$ is the outward normal vector. The jump and average operators on an exterior boundary are defined as:

$$
\begin{aligned}
& \llbracket a \rrbracket=a, \\
& \langle a\rangle=a .
\end{aligned}
$$




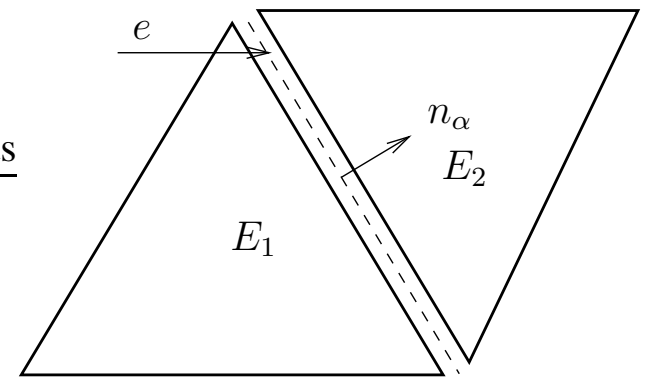

Figure 1: Neighboring elements and their shared edge.

\section{1 $C^{0}$ interior-penalty formulation}

The interior-penalty formulation for a Poisson-Kirchhoff plate has been proposed by Engel et al. [1], in which $C^{0}$ continuous basis functions are used. In the variational formulation, besides the standard part, terms have been added to weakly enforce the continuity of normal slopes on the boundaries $\Gamma^{0}$ and $\Gamma^{\theta}$ and to preserve consistency and maintain stability. The interior-penalty (IP) formulation involves: find $w^{h} \in W^{h}$ such that

$$
B_{h}\left(\bar{w}^{h}, w^{h}\right)=L_{h}\left(\bar{w}^{h}\right) \quad \forall \bar{w}^{h} \in \bar{W}^{h}
$$

where

$$
\begin{array}{r}
B_{h}\left(\bar{w}^{h}, w^{h}\right)=\int_{\Omega} \bar{w}_{, \alpha \beta}^{h} c_{\alpha \beta \gamma \delta} w_{, \gamma \delta}^{h} d \Omega-\sum_{e \in \Gamma^{0}} \int_{e}\left\langle\left(\bar{w}_{, \alpha \beta}^{h} c_{\alpha \beta \gamma \delta}\right) n_{\gamma}\right\rangle \llbracket w_{, \gamma}^{h} n_{\gamma} \rrbracket n_{\delta} d \Gamma \\
-\sum_{e \in \Gamma^{0}} \int_{e} \llbracket \bar{w}_{, \alpha}^{h} n_{\alpha} \rrbracket n_{\beta}\left\langle c_{\alpha \beta \gamma \delta} w_{, \gamma \delta}^{h} n_{\alpha}\right\rangle d \Gamma+\sum_{e \in \Gamma^{0}} \int_{e} \frac{\eta}{h} \llbracket \bar{w}_{, \alpha}^{h} n_{\alpha} \rrbracket a \llbracket w_{, \gamma}^{h} n_{\gamma} \rrbracket d \Gamma \\
-\sum_{e \in \Gamma^{\theta}} \int_{e}\left(\bar{w}_{, \alpha \beta}^{h} c_{\alpha \beta \gamma \delta}\right) n_{\gamma}\left(w_{, \gamma}^{h} n_{\gamma}\right) n_{\delta} d \Gamma-\sum_{e \in \Gamma^{\theta}} \int_{e}\left(\bar{w}_{, \alpha}^{h} n_{\alpha}\right) n_{\beta}\left(c_{\alpha \beta \gamma \delta} w_{, \gamma \delta}^{h} n_{\alpha}\right) d \Gamma \\
+\sum_{e \in \Gamma^{\theta}} \int_{e} \frac{\eta}{h}\left(w_{, \alpha}^{h} n_{\alpha}\right) a\left(\bar{w}_{, \gamma}^{h} n_{\gamma}\right) d \Gamma,
\end{array}
$$

and

$$
\begin{array}{r}
L_{h}\left(\bar{w}^{h}\right)=\int_{\Omega} \bar{w}^{h} F d \Omega-\sum_{e \in \Gamma^{\theta}} \int_{e}\left(\bar{w}_{, \alpha \beta}^{h} c_{\alpha \beta \gamma \delta}\right) n_{\gamma} g^{\theta} n_{\delta} d \Gamma+\sum_{e \in \Gamma^{M}} \int_{e} \bar{w}_{, \alpha}^{h} n_{\alpha} M d \Gamma \\
\quad+\sum_{e \in \Gamma^{Q}} \int_{e} \bar{w}^{h} Q d \Gamma+\sum_{e \in \Gamma^{\theta}} \int_{e} \frac{\eta}{h}\left(\bar{w}_{, \alpha}^{h} n_{\alpha}\right) a g^{\theta} d \Gamma,
\end{array}
$$

where $a=c_{\alpha \beta \gamma \delta} n_{\alpha} n_{\beta} n_{\gamma} n_{\delta}, \eta$ is a dimensionless penalty term and $h$ is a measure of the element size.

Consistency and conditional stability of the formulation have been proven in [1], with the formulation being stable if a large-enough penalty term is chosen. Although the IP formulation is simple, it has some drawbacks, such as the conditional stability, the loss of accuracy for large penalty values [4] and ambiguities when making the extension to nonlinear problems. In order to alleviate these difficulties, an alternative formulation is introduced in the next section. 


\subsection{Lifting-type formulation}

In work of Brezzi et al. [7], a modified version of the formulation of Bassi and Rebay [8] was proposed for second order elliptic problems. The key advantage of the formulation is that it is stable for $\eta>0$. In this section, the formulation is adapted for thin bending problems.

The formulation relies on a lifting operation on element edges. Consider first the function space $\mathcal{R}^{h}$

$$
\mathcal{R}^{h}=\left\{r_{\alpha \beta} \in L^{2}(\Omega): r_{\alpha \beta}\left(E_{i}\right) \in P^{l}\left(E_{i}\right) \forall E_{i} \in \mathcal{P}^{h}\right\} .
$$

For each element edge $e \in \Gamma^{0}, E$ denotes the union of two elements $E_{1}, E_{2} \in \mathcal{P}^{h}$ that share $e$, while for $e \in \Gamma^{\theta}, E \in \mathcal{P}^{h}$ denotes the element with edge $e$. A lifting operation is now defined by: given $u \in L^{2}(\Omega)$, find $r_{\alpha \beta}^{e}(u) \in \mathcal{R}^{h}$ such that

$$
\int_{E} v_{\alpha \beta}^{h} r_{\alpha \beta}^{e}(u) d \Omega=-\int_{e}\left\langle v_{\alpha \beta}^{h} n_{\alpha}\right\rangle \llbracket u \rrbracket n_{\beta} d \Gamma \quad \forall v_{\alpha \beta}^{h} \in \mathcal{R}^{h}
$$

Now, two functions $R_{\alpha \beta}(u)$ and $R_{\alpha \beta}^{g}(u)$ are defined as follows:

$$
\begin{aligned}
& R_{\alpha \beta}(u)=\sum_{e \in \Gamma^{0} \cup \Gamma^{\theta}} r_{\alpha \beta}^{e}(u), \\
& R_{\alpha \beta}^{g}(u)=\sum_{e \in \Gamma^{\theta}} r_{\alpha \beta}^{e}(u) .
\end{aligned}
$$

The variational equation for thin bending problems then becomes: find $w^{h} \in W^{h}$ such that

$$
B_{h}\left(\bar{w}^{h}, w^{h}\right)=L_{h}\left(\bar{w}^{h}\right) \quad \forall \bar{w}^{h} \in \bar{W}^{h}
$$

where

$$
\begin{aligned}
B_{h}\left(\bar{w}^{h}, w^{h}\right)=\int_{\Omega}\left(\bar{w}_{, \alpha \beta}^{h}+R_{\alpha \beta}\left(\bar{w}_{, \alpha}^{h} n_{\alpha}\right)\right) c_{\alpha \beta \gamma \delta}\left(w_{, \gamma \delta}^{h}+R_{\gamma \delta}\left(w_{, \gamma}^{h} n_{\gamma}\right)\right) d \Omega & \\
& +\sum_{e \in \Gamma^{0} \cup \Gamma^{\theta}} \int_{\Omega} \eta r_{\alpha \beta}^{e}\left(\bar{w}_{, \alpha}^{h} n_{\alpha}\right) c_{\alpha \beta \gamma \delta} r_{\gamma \delta}^{e}\left(w_{, \gamma}^{h} n_{\gamma}\right) d \Omega
\end{aligned}
$$

and

$$
\begin{aligned}
L_{h}\left(\bar{w}^{h}\right)=\int_{\Omega} \bar{w}^{h} F d \Omega & +\int_{\Gamma^{M}}\left(\bar{w}_{, \alpha}^{h} n_{\alpha}\right) M d \Gamma+\int_{\Gamma^{Q}} \bar{w}^{h} Q d \Gamma \\
& +\int_{\Omega}\left(\bar{w}_{, \alpha \beta}^{h}+R_{\alpha \beta}\left(\bar{w}_{, \alpha}^{h} n_{\alpha}\right)\right) c_{\alpha \beta \gamma \delta} R_{\gamma \delta}^{g}\left(g^{\theta}\right) d \Omega \\
& +\sum_{e \in \Gamma^{\theta}} \int_{\Omega} \eta r_{\alpha \beta}^{e}\left(\bar{w}_{, \alpha}^{h} n_{\alpha}\right) c_{\alpha \beta \gamma \delta} r_{\gamma \delta}^{e}\left(g^{\theta}\right) d \Omega .
\end{aligned}
$$

The functions $R_{\alpha \beta}\left(w_{, \alpha}^{h} n_{\alpha}\right)$ and $R_{\gamma \delta}\left(\bar{w}_{, \gamma}^{h} n_{\gamma}\right)$ are trial and test curvature-like functions, respectively. Jumps in the normal slope across element boundaries are 'transformed' into additional 
curvature-like terms on element interiors, see equations (20) to (22). Note also that rotation boundary conditions are enforced in the same fashion.

The lifting-type formulation has several interesting properties. As the slope jump is transformed into a curvature-type term, it is more amenable to nonlinear problems than the IP method. Like the IP method, it is consistent, but it also offers the possibility of unconditional stability. It is however more expensive computationally than the IP method due to computation of the lifting terms.

\section{NUMERICAL IMPLEMENTATION}

Besides the standard procedure used in continuous finite element methods, some additional steps are required when implementing the discontinuous Galerkin formulations. In this section, some important points when applying the above formulations for thin bending problems are presented.

In each element $E_{i} \in \mathcal{P}^{h}$, functions $w^{h}, \bar{w}^{h}$ and their derivatives can be expressed in term of nodal displacement vectors, denoted by $\boldsymbol{a}_{i}$ and $\boldsymbol{b}_{i}$, as follows:

$$
\begin{aligned}
w^{h} & =\boldsymbol{N}^{k} \boldsymbol{a}_{i}, & \bar{w}^{h} & =\boldsymbol{N}^{k} \boldsymbol{b}_{i}, \\
w_{, \alpha}^{h} & =\boldsymbol{N}_{\alpha}^{k} \boldsymbol{a}_{i}, & \bar{w}_{, \alpha}^{h} & =\boldsymbol{N}_{\alpha}^{k} \boldsymbol{b}_{i}, \\
w_{, \alpha \beta}^{h} & =\boldsymbol{N}_{\alpha \beta}^{k} \boldsymbol{a}_{i}, & \bar{w}_{, \alpha \beta}^{h} & =\boldsymbol{N}_{\alpha \beta}^{k} \boldsymbol{b}_{i},
\end{aligned}
$$

where $\boldsymbol{N}^{k}$ denote the matrix containing polynomial shape functions $P^{k}\left(E_{i}\right), \boldsymbol{N}_{\alpha}^{k}$ and $\boldsymbol{N}_{\alpha \beta}^{k}$ are matrices containing the first and the second derivatives of the shape functions, respectively. Notice equations in (28), because $w_{, \alpha \beta}^{h}$ and $\bar{w}_{, \alpha \beta}^{h}$ are symmetric second-order tensors, they can be rewritten as vectors of three components.

On each element edge $e$, let $\boldsymbol{a}_{e}$ and $\boldsymbol{b}_{e}$ denote the trial and weight nodal displacement vectors of elements $E_{1}$ and $E_{2}$ sharing $e$. Jump and average terms on $e$ are discretized as follows:

$$
\begin{aligned}
\llbracket w_{, \alpha}^{h} n_{\alpha} \rrbracket & =\boldsymbol{N}_{\mathrm{j}}^{k} \boldsymbol{a}_{e}, & \llbracket \bar{w}_{, \alpha}^{h} n_{\alpha} \rrbracket & =\boldsymbol{N}_{\mathrm{j}}^{k} \boldsymbol{b}_{e}, \\
\left\langle\left(w_{, \alpha \beta}^{h} c_{\alpha \beta \gamma \delta}\right) n_{\gamma}\right\rangle & =\boldsymbol{N}_{\mathrm{a}}^{k} \boldsymbol{a}_{e}, & \left\langle\left(\bar{w}_{, \alpha \beta}^{h} c_{\alpha \beta \gamma \delta}\right) n_{\gamma}\right\rangle & =\boldsymbol{N}_{\mathrm{a}}^{k} \boldsymbol{b}_{e},
\end{aligned}
$$

where $\boldsymbol{N}_{\mathrm{j}}^{k}$ and $\boldsymbol{N}_{\mathrm{a}}^{k}$ denote interpolation matrices for the jump of normal slopes and the average of normal bending moments on $e$, respectively. When implementing the IP formulation, by taking the above relations, the element stiffness matrix and element force vector can be formed.

When the lifting formulation is used, extra polynomial shape functions have to be used to interpolate $r_{\alpha \beta}^{e}\left(w_{, \alpha}^{h} n_{\alpha}\right), r_{\alpha \beta}^{e}\left(\bar{w}_{, \alpha}^{h} n_{\alpha}\right)$ and $v_{\alpha \beta} \in \mathcal{R}^{h}$. As equations in (28), these second order tensors are rewritten in vectors of three components. Denoting $\boldsymbol{r}_{i}, \overline{\boldsymbol{r}}_{i}$, and $\boldsymbol{v}_{i}$ respectively be vectors of the trial, the test, and an arbitrary curvature-like functions at nodes of element $E_{i}$ and $\boldsymbol{v}_{e}$ be a vector of arbitrary curvature-like functions at nodes of elements $E_{i}$ sharing $e$, we adopt the following relation:

$$
\begin{aligned}
r_{\alpha \beta}^{e}\left(w_{, \alpha}^{h} n_{\alpha}\right) & =\boldsymbol{N}^{l} \boldsymbol{r}_{i}, & r_{\alpha \beta}^{e}\left(\bar{w}_{, \alpha}^{h} n_{\alpha}\right) & =\boldsymbol{N}^{l} \overline{\boldsymbol{r}}_{i}, \\
v_{\alpha \beta}^{h} & =\boldsymbol{N}^{l} \boldsymbol{v}_{i}, & \left\langle v_{\alpha \beta}^{h} n_{\alpha}\right\rangle & =\boldsymbol{N}_{\mathrm{a}}^{l} \boldsymbol{v}_{e},
\end{aligned}
$$

where $\boldsymbol{N}^{l}$ and $\boldsymbol{N}_{\mathrm{a}}^{l}$ are the interpolation matrices containing polynomial functions $P^{l}\left(E_{i}\right)$. Im- 
plementing lifting operations (20) to (23) leads to the following relations:

$$
\begin{aligned}
r_{\alpha \beta}^{e}\left(w_{, \alpha}^{h} n_{\alpha}\right) & =-\boldsymbol{N}^{l}\left(\int_{E} \boldsymbol{N}^{l^{T}} \boldsymbol{N}^{l} d \Omega\right)^{-1} \int_{e} \boldsymbol{N}_{\mathrm{a}}^{l^{T}} \boldsymbol{N}_{\mathrm{j}}^{k} d \Gamma \boldsymbol{a}_{e}, \\
r_{\alpha \beta}^{e}\left(\bar{w}_{, \alpha}^{h} n_{\alpha}\right) & =-\boldsymbol{N}^{l}\left(\int_{E} \boldsymbol{N}^{l^{T}} \boldsymbol{N}^{l} d \Omega\right)^{-1} \int_{e} \boldsymbol{N}_{\mathrm{a}}^{l^{T}} \boldsymbol{N}_{\mathrm{j}}^{k} d \Gamma \boldsymbol{b}_{e}, \\
r_{\alpha \beta}^{e}\left(g^{\theta}\right) & =-\boldsymbol{N}^{l}\left(\int_{E} \boldsymbol{N}^{l^{T}} \boldsymbol{N}^{l} d \Omega\right)^{-1} \int_{e} \boldsymbol{N}_{\mathrm{a}}^{l^{T}} g^{\theta} d \Gamma .
\end{aligned}
$$

An important point that should be noted is the choice of lifting shape functions $P^{l}\left(E_{i}\right)$. In general, the order $l$ is arbitrary. However, numerical results shown that when adopting shape functions $P^{k}\left(E_{i}\right)$ of the order $k=2$ for $w$, using lifting functions of the order $l=k-2$ leads to good results, while with $k=3, l$ can belongs a larger range, that is $l=k, k-1$ or $k-2$.

The global stiffness matrix for the IP formulation is sparser than for the lifting formulation. This derives from the fact that while the additional integration on the edges for the IP formulation couples neighboring elements, the lifting operator couples all elements surrounding a given element [7].

\section{NUMERICAL EXAMPLES}

A square plate with a side length $L=2 \mathrm{~m}$ and thickness $t=0.01 \mathrm{~m}$ is considered, and Young's modulus $E=10^{8} \mathrm{~N} / \mathrm{m}^{2}$ and Poisson's ratio $\nu=0.3$. Two cases are examined: simple support $\left(\Gamma^{w}=\Gamma, \Gamma^{M}=\Gamma, g^{w}=0, M=0\right)$ and clamped support $\left(\Gamma^{w}=\Gamma, \Gamma^{\theta}=\Gamma, g^{w}=0\right.$, $g^{\theta}=0$ ). A uniform distributed force $F=10 \mathrm{~N} / \mathrm{m}^{2}$ applied. Series solutions for the models can be found in [9, pages 197-202]. Defining $D=E t^{3} / 12\left(1-\nu^{2}\right)$, the maximum deflection for simply supported case $w_{s}^{\max }=0.00406 F L^{4} / D=0.0355 \mathrm{~m}$ and for clamped support case $w_{c}^{\max }=0.00126 F L^{4} / D=0.0110 \mathrm{~m}$. We employ quadratic $(k=2)$ and cubic $(k=3)$ triangular elements with uniform meshes, and examine both the IP and lifting formulations. For the lifting formulation, lifting shape functions of the order $l=k-2$ are used.

For the simply supported case, the $L_{2}$ norm of the error is calculated. A convergence rate of order two can be observed when employing quadratic basis functions, as seen in figures 2 and 3 and of order four is observed when using cubic basis functions, as seen in figures 4 and 5 , for both the IP and lifting formulations. However, there exists an unstable result with a low value of $\eta$ for the IP formulation, which is evident in figures 2 and 4 where the line corresponding to $\eta=1$ is not straight. There is no such problem for the lifting formulation in figures 3 and 5 The critical value of $\eta$ for stability is not easy to determine for the IP formulation, but in the case of lifting formulation it appears that $\eta>0$ guarantees stability. This remains to be proven for the presented formulation. The results also shows that, in general, the larger value of $\eta$ the larger the error, especially when quadratic shape functions are used.

For the clamped case, normalized maximum displacements are presented in figures 6 and 7 for quadratic elements for the IP and lifting formulations, respectively, and in figures 8 and 9 for cubic elements, again for the IP and lifting formulations, respectively. There is a locking problem with a high value of $\eta$ when using quadratic shape functions with full integration on interior boundaries (two points on each edge, $p=2$ ). A reduced integration with one per edge, $p=1$, leads better results, as shown in figures 10 and 11 in which the locking problems have been alleviated. 


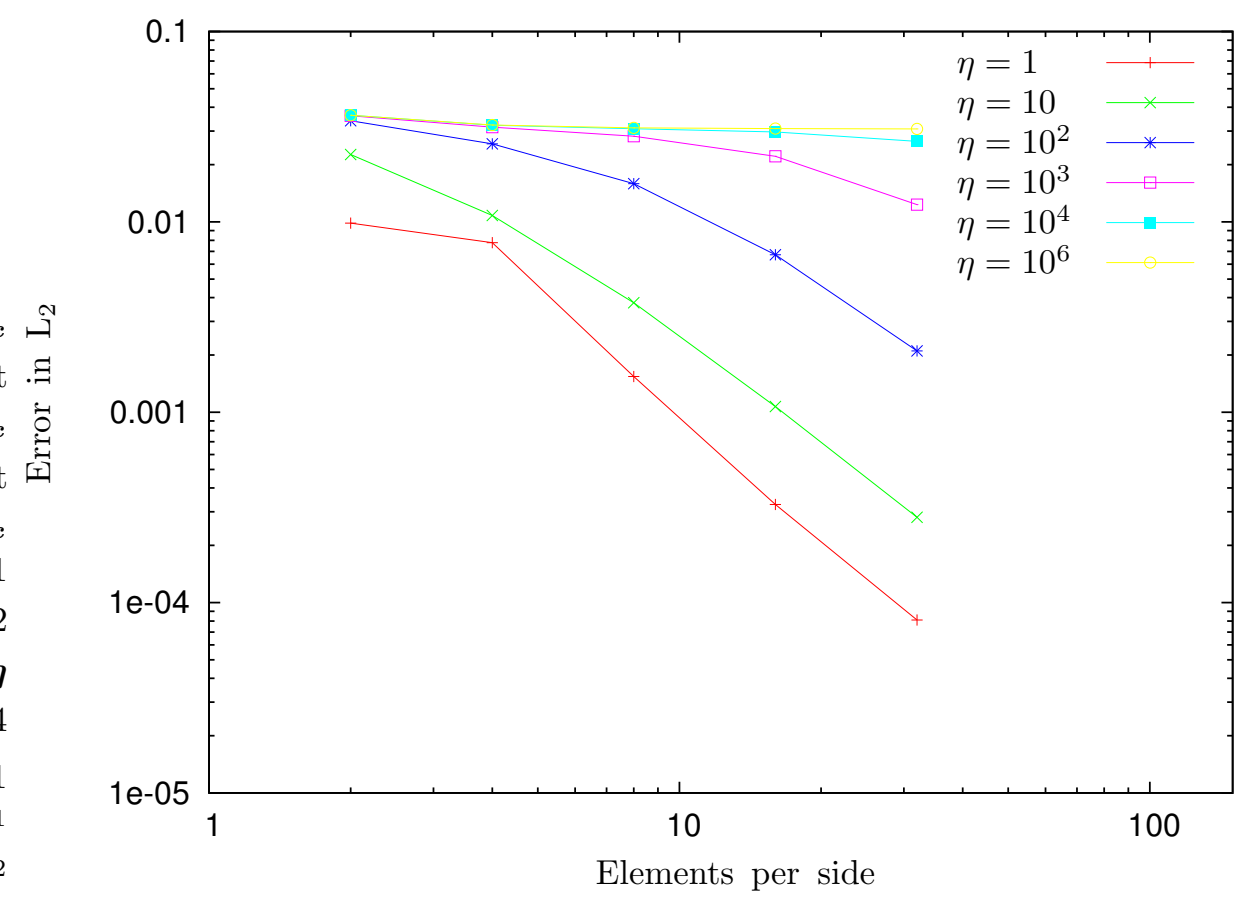

Figure 2: Convergence of simply supported square plate using the IP formulation and quadratic shape functions.

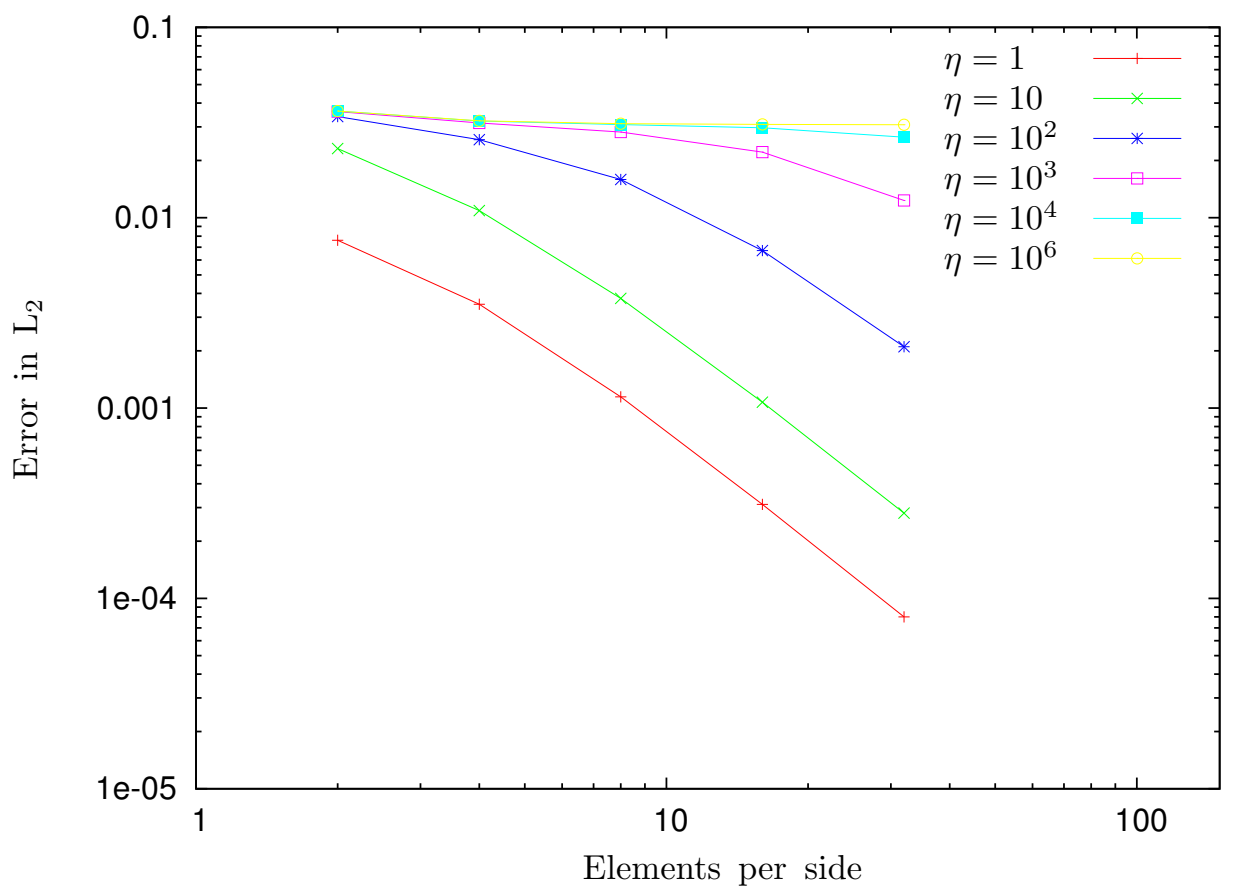

Figure 3: Convergence of simply supported square plate using the lifting formulation and quadratic shape functions. 


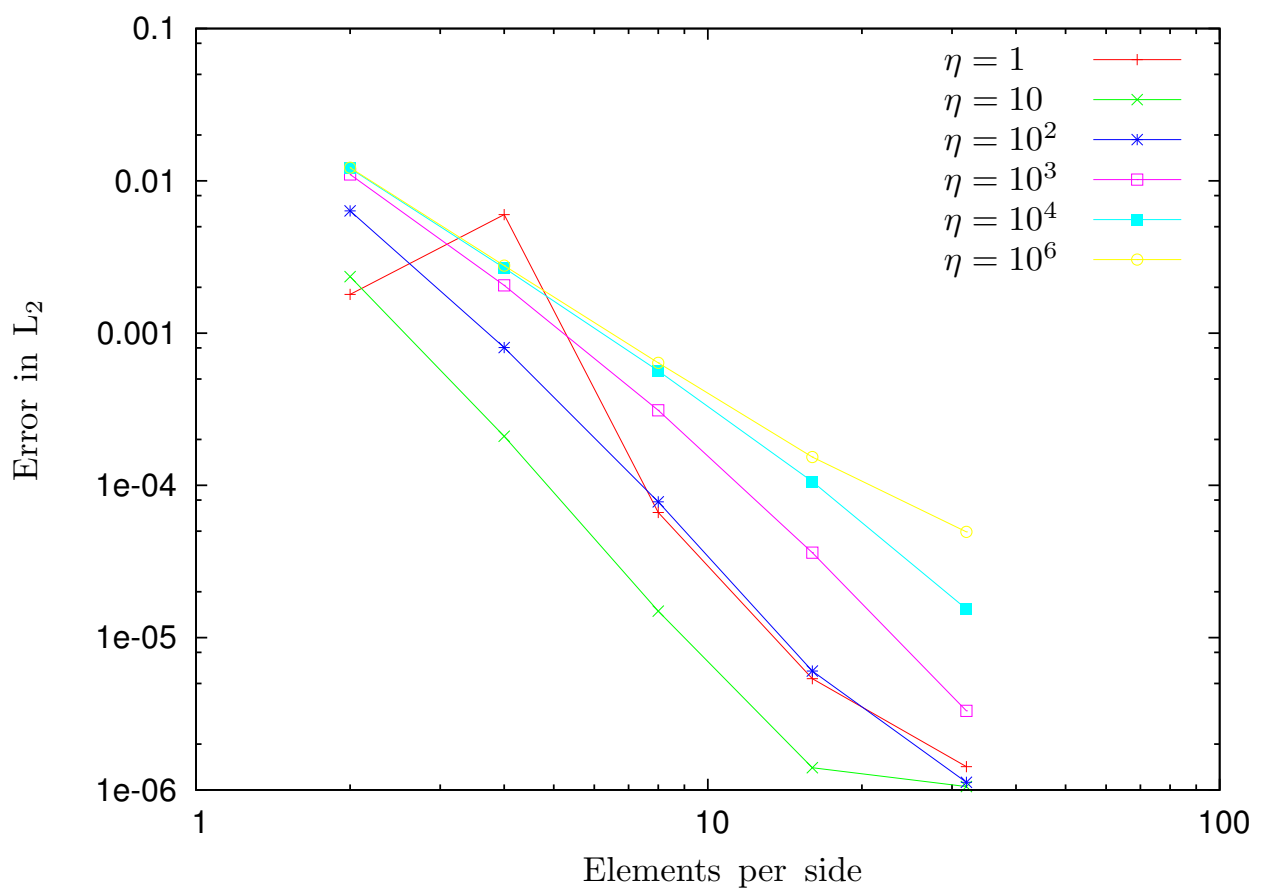

Figure 4: Convergence of simply supported square plate using the IP formulation and cubic shape functions.

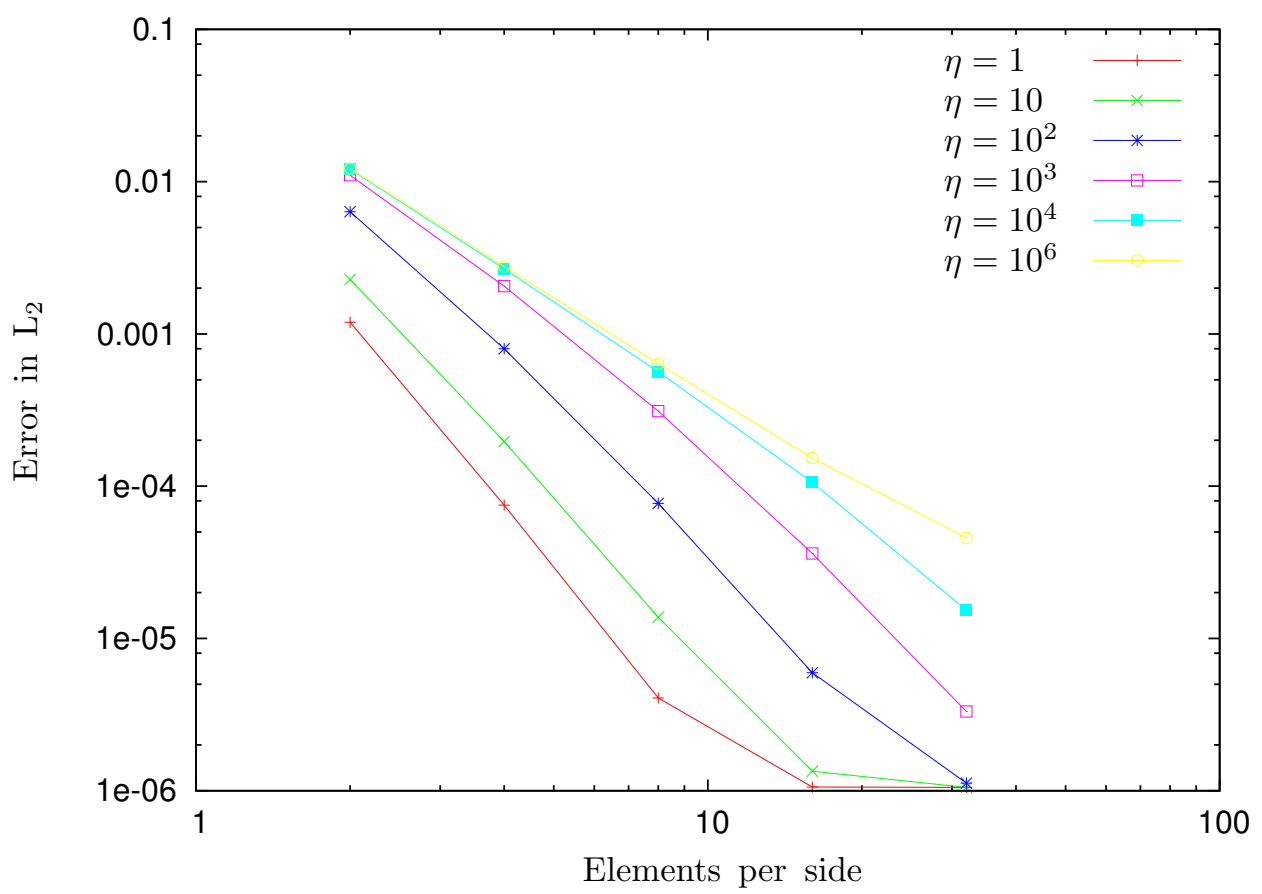

Figure 5: Convergence rate of simply supported square plate using the lifting formulation and cubic shape functions. 


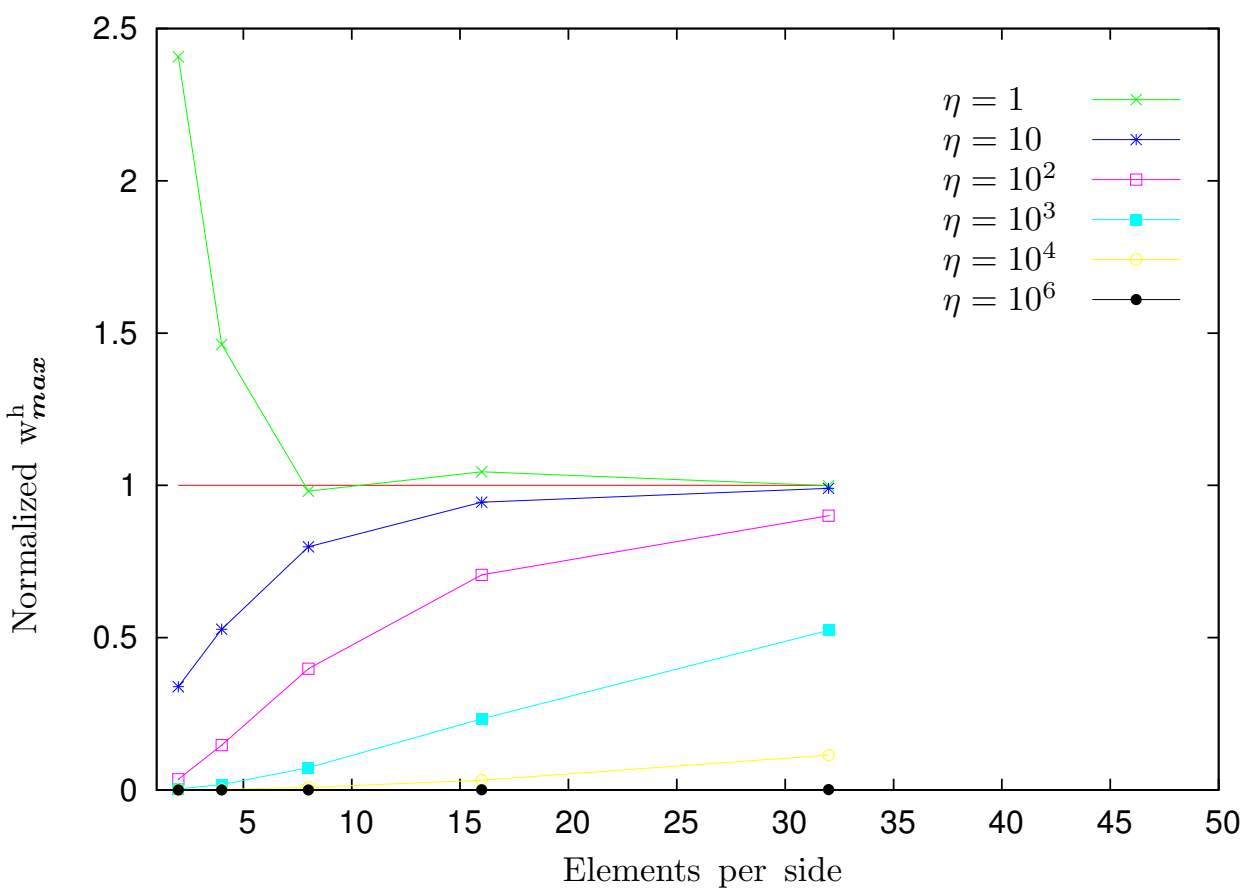

Figure 6: Normalized maximum deflection of clamped square plate using the IP formulation and quadratic shape functions.

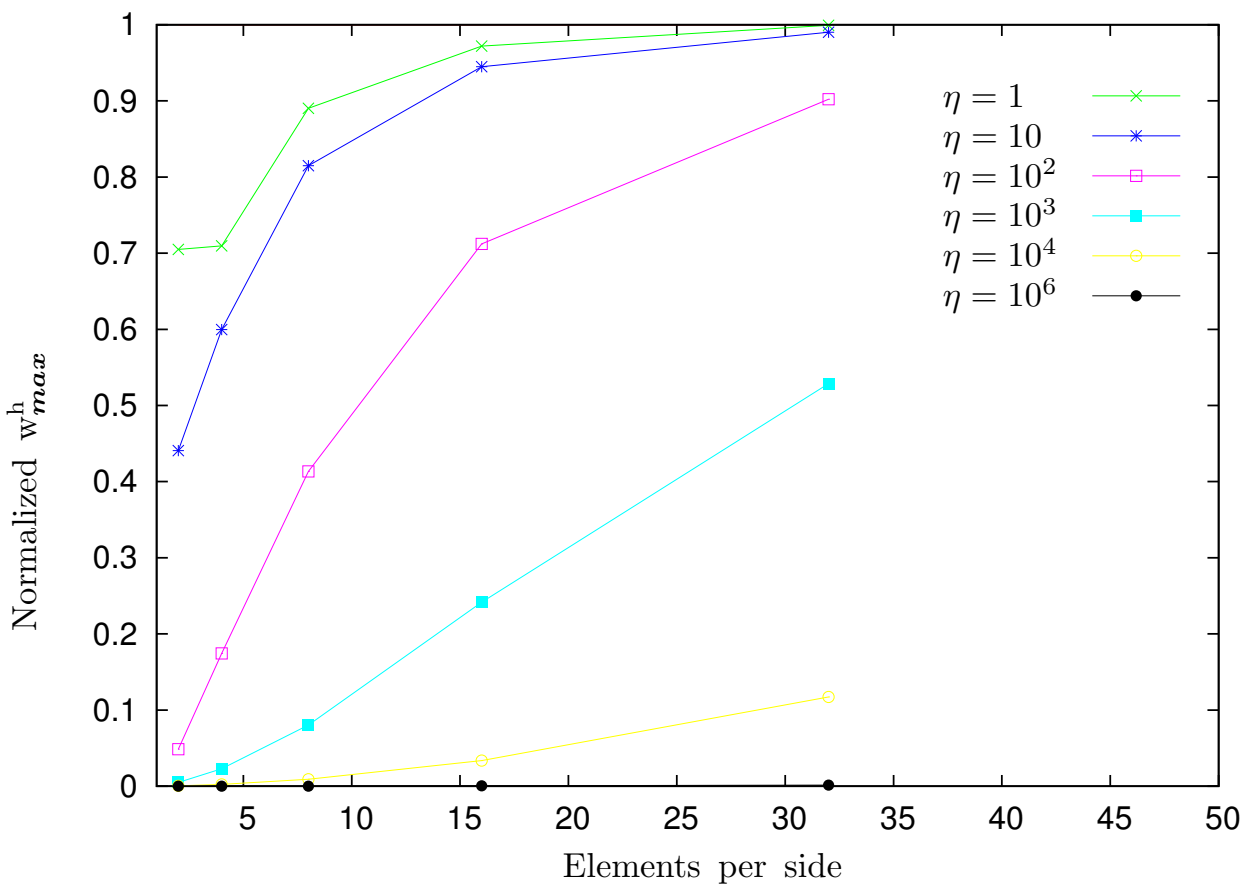

Figure 7: Normalized maximum deflection for clamped square plate using the lifting formulation and quadratic shape functions. 


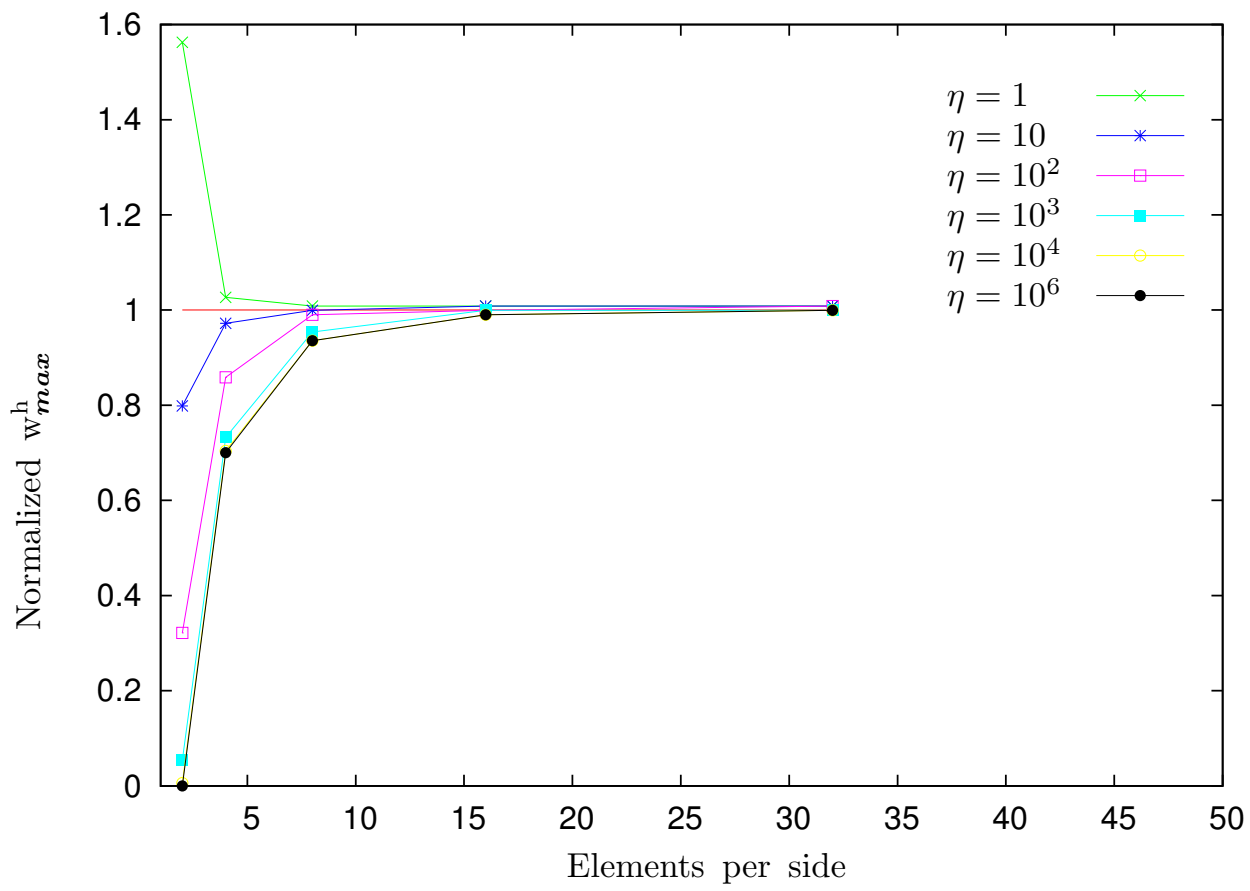

Figure 8: Normalized maximum deflection of clamped square plate using the IP formulation and cubic shape functions.

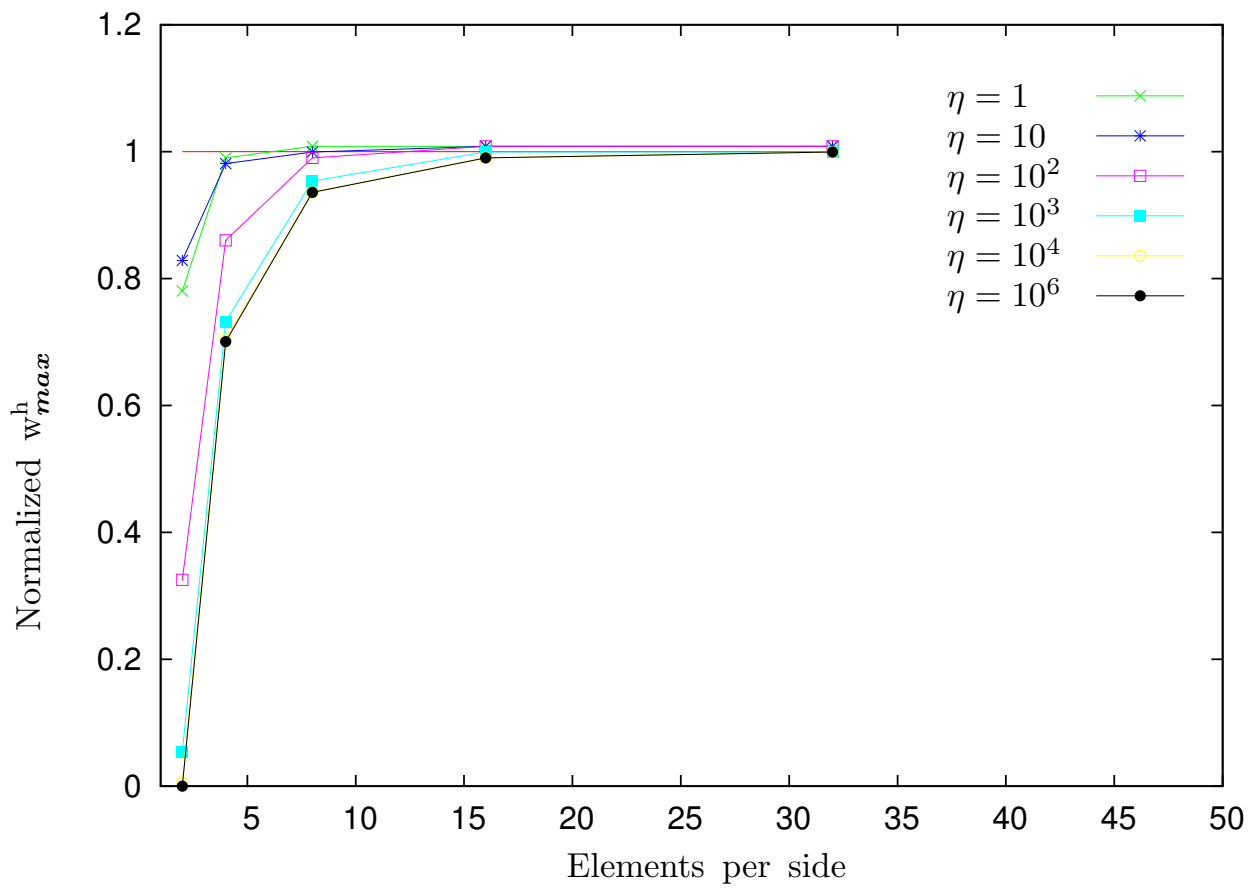

Figure 9: Normalized maximum deflection for clamped square plate using the lifting formulation and cubic shape functions. 


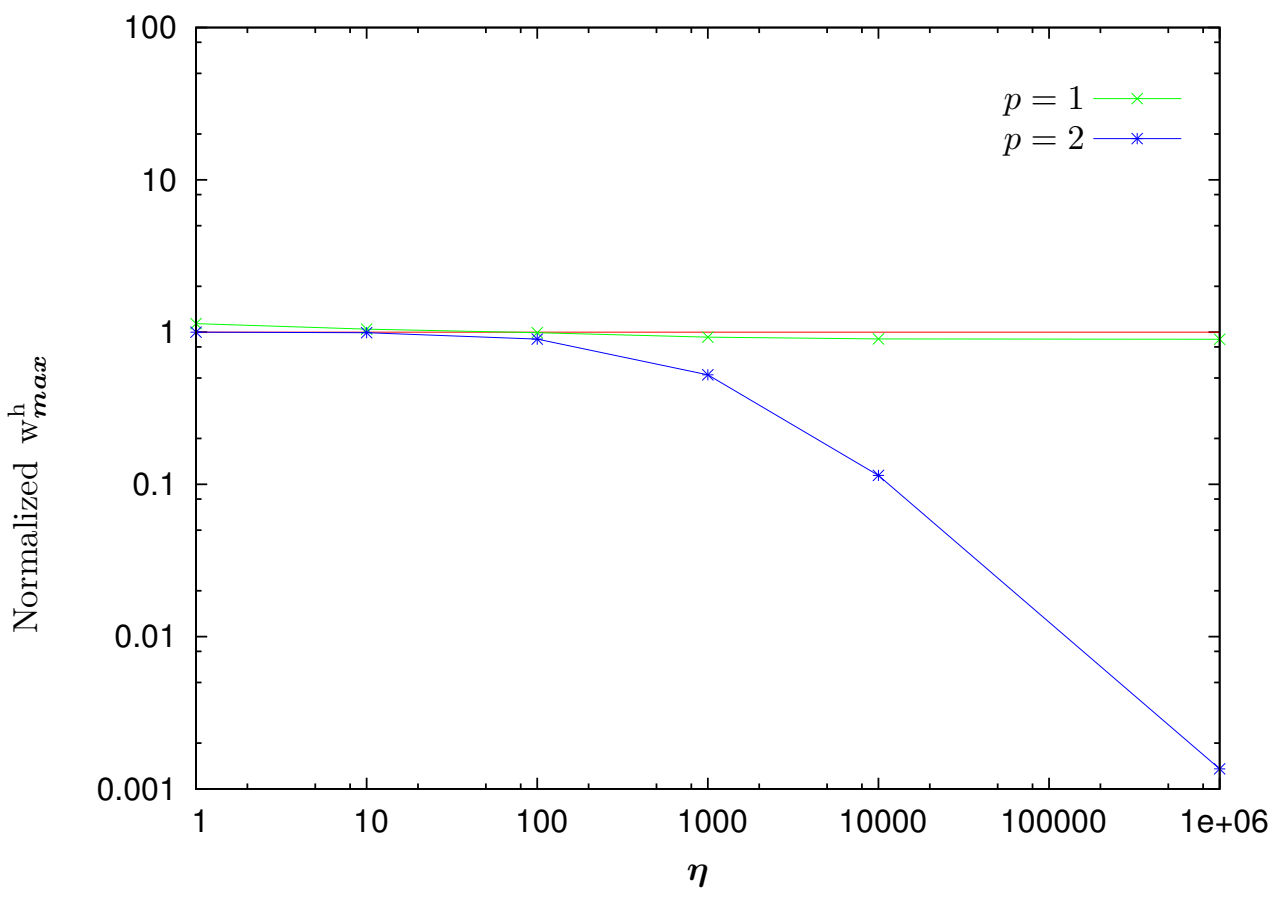

Figure 10: Normalized maximum deflection for clamped square plate using the IP formulation, quadratic shape functions, with 2048 elements and one integration point each edge

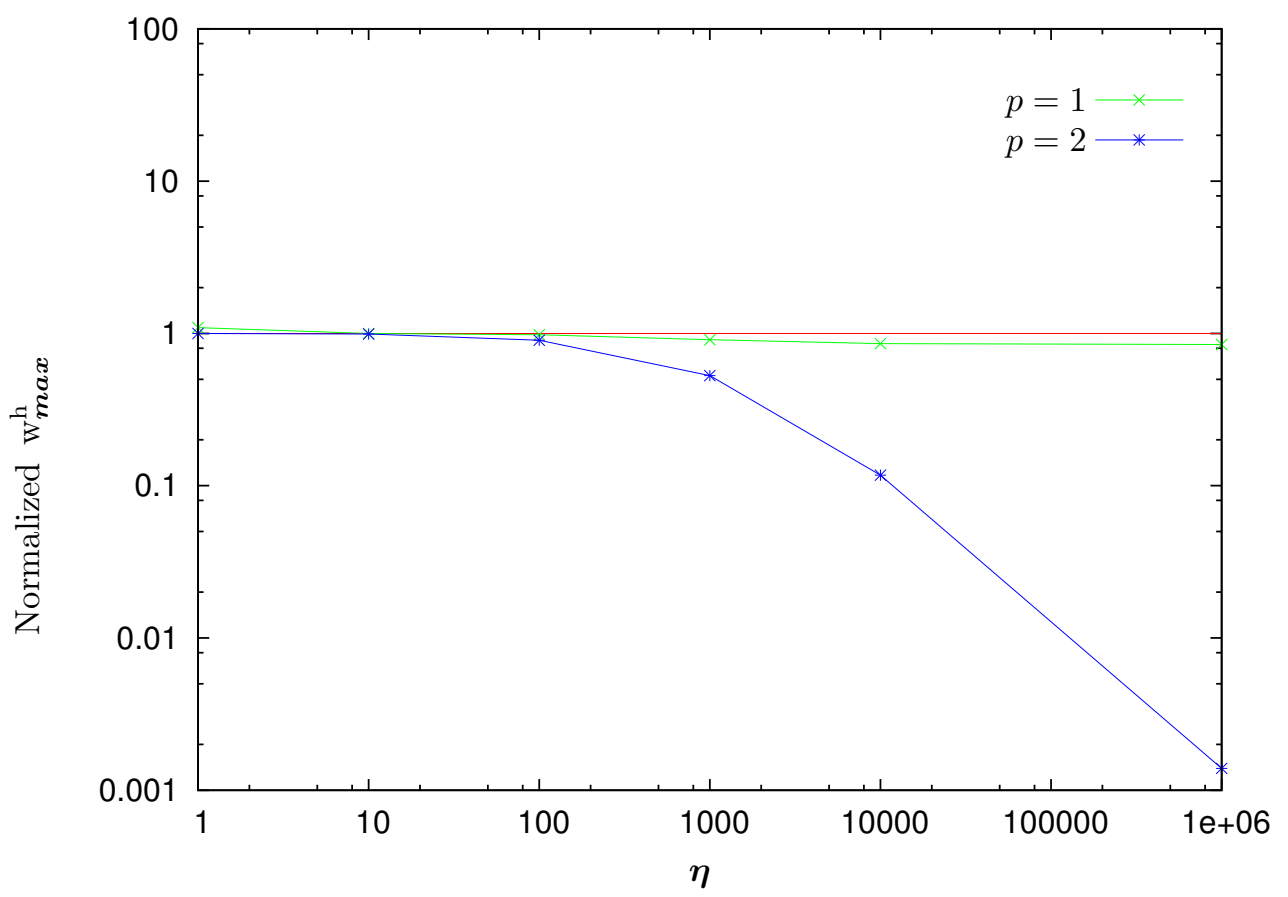

Figure 11: Normalized maximum deflection for clamped square plate using the lifting formulation, quadratic shape functions, with 2048 elements and one integration point each edge 


\section{CONCLUSIONS}

A single-field discontinuous Galerkin method for modelling thin plates using $C^{0}$ shape functions has been presented. It derives from work on discontinuous Galerkin methods for secondorder equations. The jump in the normal derivative is 'lifted', converting it to an effective curvature within elements. Numerical results indicate that the method is stable for any penalty parameter greater than zero, and the rate of convergence is optimal for cubic elements. In contrast to the interior penalty-type method, the method is slightly more complex to implement, with the reward being the likely unconditional stability. Also, it lends itself better for application to nonlinear problems. Outstanding issues include proving stability through a priori analysis of the method, and an analytical examination of the convergence behavior.

\section{Acknowledgments}

Financial support from the Vietnamese Ministry of Education and Training (VMOET) and the TuD Management Centre for International Cooperation (CICAT) for the first author is gratefully acknowledged.

\section{References}

[1] G. Engel, K. Garikipati, T. J. R. Hughes, M. G. Larson, L. Mazzei, and R. L. Taylor. Continuous/discontinuous finite element approximations of fourth-order elliptic problems in structural and continuum mechanics with applications to thin beam and plates, and strain gradient elasticity. Computer Methods in Applied Mechanics and Engineering, 191:36693750, 2002.

[2] G. N. Wells, K. Garikipati, and L. Molari. A discontinuous galerkin formulation for a strain gradient-dependent damage model. Computer Methods in Applied Mechanics and Engineering, 193:3633-3645, 2004.

[3] L. Molari, G. N. Wells, K. Garikipati, and F. Ubertini. A discontinuous galerkin method for strain gradient-dependent damage: Study of interpolations, convergence and two dimensional problems. Computer Methods in Applied Mechanics and Engineering, 195: 1480-1498, 2006.

[4] S. C. Brenner and L. Y. Sung. $\mathrm{C}^{0}$ interior penalty methods for fourth order elliptic boundary value problems on polygonal domains. Journal of Scientific Computing, 22(1):83-118, 2005.

[5] D. N. Arnold. An interior penalty finite element method with discontinuous elements. SIAM Journal on Numerical Analysis, 19:742-760, August 1982.

[6] D. N. Arnold, F. Brezzi, B. Cockburn, and L. D. Marini. Unified analysis of discontinuous galerkin methods for elliptic problems. SIAM Journal on Numerical Analysis, 39(5):17491779, 2002.

[7] F. Brezzi, G. Manzini, D. Marini, P. Pietra, and A. Russo. Discontinuous galerkin approximations for elliptic problems. Numer. Methods Partial differential Equations, 16:365-378, 2000 . 
[8] F. Bassi and S. Rebay. Numerical evaluation of two discontinuous galerkin methods for the compressible navier-stokes equations. International Journal for Numerical Methods in fluids, 40:197-207, 2002.

[9] S. P. Timoshenko and S. W. Krieger. Theory of Plates and Shells. McGraw-Hill, 1989. 